\title{
Design and Implementation of Graphical User Interface of Digital STB
}

\author{
FANG Rong ${ }^{1}$ \\ ${ }^{1}$ JINHUA POLYTECHNIC \\ zhejiang jinhua,china,321017 \\ Email:9258724@qq.com
}

\author{
HOU Meng-shu ${ }^{2}$ \\ ${ }^{2}$ University of Electronic Science and Technology,sichuan \\ chengdu,china, 611731 \\ mshou@uestc.edu.cn
}

\begin{abstract}
Set-Top-Box(STB) as the background, through an understanding of developing environment of graphical user interface (GUI), To have a general design of STB GUI system, and To realize the STB GUI, and give a realized example of building an GUI under this system.
\end{abstract}

Keywords-Graphical User Interface; Embedded System; Set-Top-Box

\section{Introduction}

Along with the rapid development of information technology and digital technology, the popularity of digital TV will surely become the future development trend of the TV industry. Digital TV STB is a kind of typical embedded equipment. In recent years, embedded equipment become increasingly widespread use of the embedded system that have developed rapidly in People's Daily life. As embedded device hardware performance continues to improve, Consumers, especially for high performance function embedded man-machine interactive interface requirements are more and more urgent. Including of smart phone, PDA, STB, more and more embedded system Require full-featured GUI system support. Therefore, the key technology for embedded systems - the research of embedded GUI technology has important practical significance.

\section{Development environment introduction}

This paper use the assembly instruction set based on the Mcore, use the ANSI C for code development, use CodeWarrior for Mcore R2.0 compiler, compile .S format file. Use GxUpDataFlash code downloader, support .S、.BIN format file; downloader can generate .BIN format Flash programmer programming file. Use FontCreaterV1.1 character font generation tool to generate the system including the standard $24 \times 24$ Chinese, $16 \times 16$ ASCII font and other fonts used in the system, including French, German, Russian and other foreign characters and $20 \times 20$ Chinese small fonts. Use BmpPacketPath gallery generation tool to generate system uses icons and compression pictures. Use BMP2YUV generation tool to generate the .BIN format boot screen. Hardware core of Software development use Gx6101 integrated chip. Gx6101 is the first highly integrated digital satellite receiver and decoding system chip, the internal integration of the channel ADC、 video DAC、DVB-S demodulator - decoder, 32-bit high-performance embedded CPU, MPEG2-TS streaming solution multiplexer, MPEG2 video and audio decoder, video de-interlacing and post-processing unit, OSD generator and TV encoder modules for digital satellite receivers to provide a more competitive machine program.

\section{The design of STB GUI}

Through the analysis of STB functionality, GUI development library system needs to implement the module is divided into three categories: graphics output class, device output class and window object. Graphics output class includes regional operations, basic graphics operations and bitmap operations. Device output class contains infrared remote control input and timer input. Window object consists mainly of dialog boxes, menus and controls.

Major elements of interface design are:

(1) Text box: used to display messages, generally do not support any operation. Support functions that operate on the text box include drawing lines, rectangles, filled rectangles, and display the string.

(2) Button: at the interface shows a similar "button" graphics, mainly for the user to select operation. From the visual effect , the "button" to press or bounce. In programming, we use the two images are displayed alternately, so that the button has dynamic effects. For example,GxDraw3Dbutton function: used to draw a 3D button.

(3) Dialog box: mainly used to finalize the operation selected by the user, specify the location of display the specified width and height. This element is actually composed of button and text box. For example, GxDrawSelectMsgBox function: used to draw the dialog with OK and Cancel.

(4)Input text box: mainly used to input data, such as adjusting the satellite's parameters, and so on.

\section{STB GUI Implementation}

A

\section{Set specific keys}

Commonly used keys: $0 \sim 9$, ${ }^{*}, \#$, down, up,left, right, OK, Cancel (exit / Delete key). in the mode of off menu, press the "OK" button brings up the main menu. Have the selection function of getting into the sub-menu and confirm. Press "Cancel" button to return to the previous menu or used as a backspace button when enter text and numbers. When in the text input mode of exit the level menu, you can continuously press the key of 1.5 to 2 seconds.

The following are commonly used in remote control function key names and their code values:

$\begin{array}{llc}\text { Key name } & \text { Code value } & \text { function keys } \\ \text { PowerKey } & \text { 0xde } & \text { remote control switch } \\ \text { UpKey } & \text { 0x7e } & \text { up key }\end{array}$




$\begin{array}{lcc}\text { DownKey } & \text { 0xee } & \text { down key } \\ \text { RightKey } & \text { 0xbe } & \text { right key } \\ \text { LeftKey } & \text { 0xf6 } & \text { left key } \\ \text { MenuKey } & \text { 0x36 } & \text { open the menu } \\ \text { OKKey } & \text { 0x66 } & \text { OK button } \\ \text { RemoteNum0 } & \text { 0xb6 } & \text { number key 0 } \\ \text { RemoteNum1 } & \text { 0x1e } & \text { number key 1 } \\ \text { RemoteNum2 } & \text { 0x9e } & \text { number key 2 } \\ \text { RemoteNum3 } & \text { 0x5e } & \text { number key } 3 \\ \text { RemoteNum4 } & \text { 0x2e } & \text { number key } 4 \\ \text { RemoteNum5 } & \text { 0xae } & \text { number key } 5 \\ \text { RemoteNum6 } & \text { 0x6e } & \text { number key } 6 \\ \text { RemoteNum7 } & \text { 0x0e } & \text { number key } 7 \\ \text { RemoteNum8 } & \text { 0x8e } & \text { number key } 8 \\ \text { RemoteNum9 } & \text { 0x4e } & \text { number key 9 } \\ \text { RemoteInfo } & \text { 0xc6 } & \text { display information } \\ \text { RemoteTvBrodcast } & \text { 0x26 } & \text { television broadcasting } \\ \text { RemoteVolumChannel } & \text { 0x16 } & \text { channel selection } \\ & \end{array}$

$\begin{array}{lcl}\text { RemoteHorn } & \text { 0xd6 } & \text { mute } \\ \text { RemoteNinePicture } & \text { 0xa6 } & \text { nine pictures } \\ \text { RemoteExit } & \text { 0x96 } & \text { exit key } \\ \text { RemotePause } & \text { 0xdd } & \text { pause key } \\ \text { SelectSatellite } & \text { 0x2f } & \text { select satellite }\end{array}$

\section{B Program flow}

The entire user interface design process flow are as follows: After system initialization, create a task, the task will be established to receive user's button input from the remote control in the beginning to the end, then depending on the key value to select the information delivered to the appropriate tasks. If the user presses the number keys on the remote control, the digital message transfer to the channel number to display task, not only channel information on-screen with the user's input has changed, but by calling the appropriate function, the system switch to the corresponding channel. If the user presses the menu button on the remote control, the system passes the message to the main menu to display the task, and then into the sub-menu keys to show the task.

(1) The main task of the user interface flow chart is shown in Figure 1.

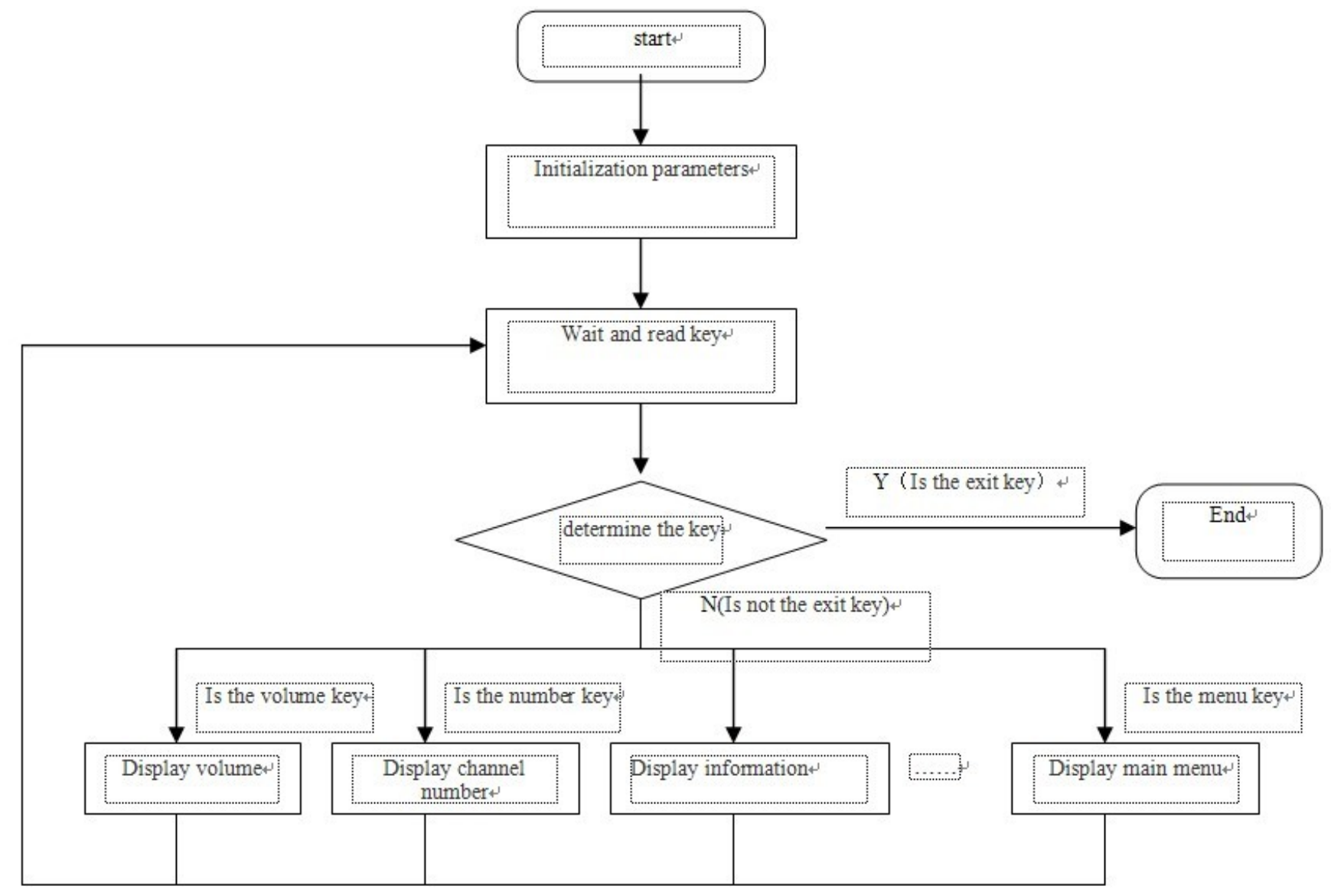

Figure 1 the main task of the user interface flow diagram

(2)In the user's option, from the main menu to enter the submenu at all levels. Each menu must first set their own data to determine the realization form, and then in turn call the menu of the constituent elements of the display function 
to display the menu, read some of the remote receiver to send a message queue key value, then according to the corresponding callback function to achieve the menu functions. The menu is a part of the process step by step from the main program to the subroutine nesting process. Process is shown in Figure 2.

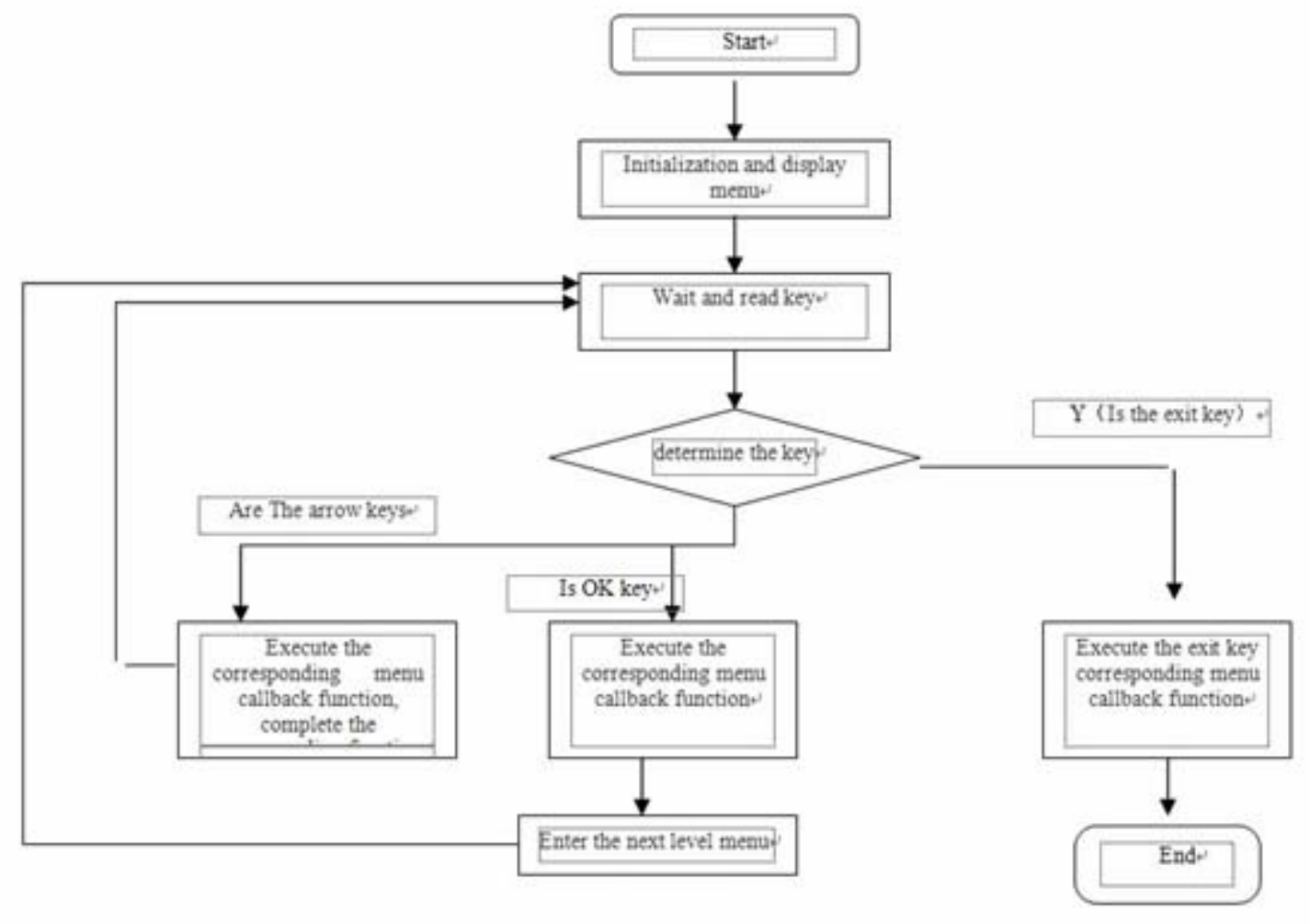

Figure 2 menu shows the task flow chart

\section{User interface realization example}

Here is a simple HelloWorld interface development instance diagram (Figure 3).

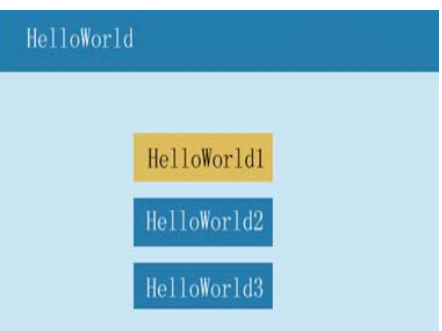

Figure 3 HelloWorld interface development example

\section{Conclusion}

This paper combined with the design and development of new digital STB products, on the basis of the research and analysis of embedded GUI systems, focusing on the development and implementation of digital STB GUI module, through a lot of work and effort, the results achieved have been put into practical use. In actual use, the GUI platform designed to have a good effect, and fully meet the actual needs of the project.

\section{References}

[1] Li Ying, Gan Yuyu. The research and design of New digital set-top-box of the GUI system [J]. Information terminal, 2007,31 (5) :40-42.

[2] LIU Lian-hao, Zheng Wei.Based on embedded platform network video system [J]. Computer and Information Technology, 2010 (01).
[3] Fu Qi, Yang Fei, Jiang Jian, Chen Wei, MultiBus-CPU module design based on embedded technology [J]. Microcontroller and Embedded Systems, 2010, (05).

[4] [4] Jack Ganske.Changes in Embedded Design Method Technology.USA:Embedded systems Programming, 2004: 47-56.

[5] [5] Ken Klask.Guide to GUI[J],Appliance Design,2005,53(7):10-13.

[6] [6] Charles Poynton,Digital video and HDTV algorithms and interface,Morgan Kaufmann Publishers,2003:7-23.

[7] [7] Memon A M. GUI testing:Pitfalls and Proeess.Computer, 2002, No.8, Vol.35, 87-88.

[8] [8] Haraikawa $\mathrm{T}$, Sakamoto $\mathrm{T}$ Hase $\mathrm{T}$, et al.A framework for GUI-based remote operation of home appliances through Power-line communication.Consumer Electronies, 2002.ICCE.2002 Digest of Technical Papers.International Conference on, 2002, 184-185.

[9] [9] Ben Shneiderman.Designing the User Interface Strategies for Effective Human-Computer Interaction Third Edition,Beijing:Publishing House of Electronies Industry, 2004, 5-11.

[10] [10] Graunke P T, Krishnamurthi S.Advanced control flows for flexible graphical user interfaces or growing GUIs on trees or bookmarking GUIs.Software Engineering 2002.ICSE2002.Proceedings of the 24rd International Conference on, 2002, 277-287. 